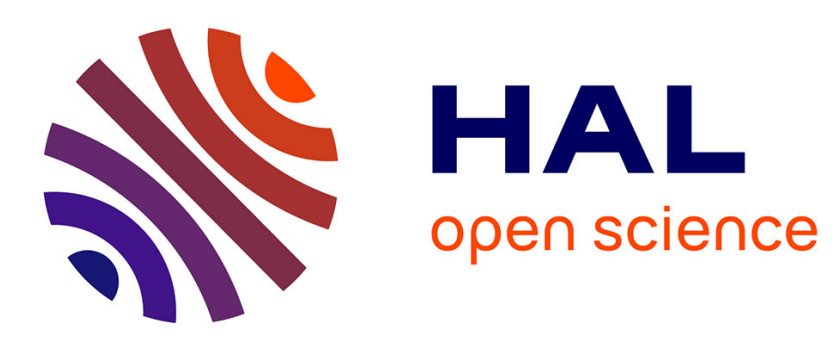

\title{
A Multifractal-based Wavefront Phase Estimation Technique for Ground-based Astronomical Observations
}

\author{
Suman Kumar Maji, Hussein M. Yahia, Thierry Fusco
}

\section{To cite this version:}

Suman Kumar Maji, Hussein M. Yahia, Thierry Fusco. A Multifractal-based Wavefront Phase Estimation Technique for Ground-based Astronomical Observations. IEEE Transactions on Geoscience and Remote Sensing, 2015, 99, pp.11. 10.1109/TGRS.2015.2487546 . hal-01254482

\section{HAL Id: hal-01254482 \\ https://hal.inria.fr/hal-01254482}

Submitted on 12 Jan 2016

HAL is a multi-disciplinary open access archive for the deposit and dissemination of scientific research documents, whether they are published or not. The documents may come from teaching and research institutions in France or abroad, or from public or private research centers.
L'archive ouverte pluridisciplinaire HAL, est destinée au dépôt et à la diffusion de documents scientifiques de niveau recherche, publiés ou non, émanant des établissements d'enseignement et de recherche français ou étrangers, des laboratoires publics ou privés. 


\title{
A Multifractal-based Wavefront Phase Estimation Technique for Ground-based Astronomical Observations
}

\author{
Suman Kumar Maji, Hussein M. Yahia and Thierry Fusco
}

\begin{abstract}
Turbulence in the Earth's atmosphere interferes with the propagation of planar wavefronts from outer space resulting in a phase distorted non-planar wavefront. This phase distortion is responsible for the refractive blurring of images accounting to the loss in spatial resolution power of ground-based telescopes. The technology widely used to remove this phase distortion is Adaptive Optics (AO). In AO, an estimate of the distorted phase is provided by a wavefront sensor (WFS) in the form of low-resolution slope measurements of the wavefront. The estimate is then used to create a corrected wavefront, that (approximately) removes the phase distortion from the incoming wavefronts. Phase reconstruction from WFS measurements is done by solving large linear systems followed by interpolating the low-resolution phase to its desired high-resolution. In this paper, we propose an alternate technique to wavefront phase reconstruction using concepts derived from the Microcanonical Multiscale Formalism (MMF), which is a specific approach to multifractality. We take into account an a priori information of the wavefront phase, provided by the multifractal exponents. Then through the framework of multiresolution analysis and wavelet transform, we address the problem of phase reconstruction from low-resolution WFS measurements. Comparison, in terms of reconstruction quality, with classical techniques in $\mathrm{AO}$ proves the superiority of our approach.
\end{abstract}

\section{Index Terms}

Suman Kumar Maji and Hussein Yahia are with the Geostat team (Geometry and Statistics in Acquisition Data: http://geostat.bordeaux.inria.fr) of INRIA, 200 rue de la Vieille Tour, 33405 Talence Cedex, France (Email: sumankumar.maji@inria.fr; hussein.yahia@inria.fr).

Thierry Fusco is with the Department of Theoretical \& Applied Optics (DOTA/E) of ONERA, Chemin de la Huniére, BP 80100, 91123 Palaiseau Cedex, France (Email: thierry.fusco@onera.fr). 
wavefront phase reconstruction, adaptive optics, multifractal exponents, multiresolution analysis, wavelets.

\section{INTRODUCTION}

Light from a distant spatial object before entering the Earth's atmosphere is a planar wavefront. Turbulence in the atmosphere causes refractive index variations that interfere with the propagation of light through this medium. As a result, the phase of a planar wavefront gets spatially modified as the wavefront gets distorted while travelling through this medium. The resultant complex field arising out of turbulence exhibits random fluctuations in its phase $\phi$ and can be expressed mathematically, at the telescope pupil, as [1]:

$$
\Psi(\vec{x})=A(\vec{x}) \exp [i \phi(\vec{x})]
$$

where $A(\vec{x})$ is the amplitude of the resultant field and $\vec{x} \equiv(x, y)$ are the coordinates in the telescope pupil. The image of the observed spatial object thus formed is degraded in resolution and blurred [1], [2], [3]. A typical example of wavefront distortion due to atmospheric turbulence and its effect on image formation in ground-based astronomy is shown in Fig 1a and Fig 1b.

In turbulence affected imaging blurring therefore occurs due to dynamic random deformations in the wavefront. These deformations defocus long exposures and blurring turns out to be very difficult to quantify (or even define objectively) in general image processing terms. One way to typically characterize blurring effects is given by the imaging systems point spread function (PSF). The PSF describes the response of an imaging system to a point source or point object and characterizes atmospheric blurring effects that are spatially invariant in the immediate field of view. The resultant image is therefore the PSF of the telescope + atmosphere optical system. The PSF is generally expressed in terms of the distorted wavefront phase $\phi$ as :

$$
p_{\phi}(\vec{x})=\left|\mathcal{F}^{-1}\{P(\vec{x}) \exp [i \phi(\vec{x})]\}\right|^{2},
$$

where $\mathcal{F}$ denotes the 2-D Fourier transform and $P(\vec{x})$ denotes the pupil (or aperture) function of the telescope i.e. is 1 inside the pupil and 0 otherwise. The goal of any AO system is to remove the phase error $\phi$ from the incoming wavefronts ${ }^{1}$ (i.e. the effect of atmosphere optical system).

\footnotetext{
${ }^{1}$ It should be noted here that although AO is the preferred application, other solutions like space based telescopes (Ex: The Hubble telescope. They provide a complete solution but are very hard to set, maintain, upgrade and support), image postprocessing for Earth-based telescopes with lucky imaging techniques also exist.
} 
If done exactly, the resulting PSF then has the form:

$$
p_{0}(\vec{x})=\left|\mathcal{F}^{-1}\{P(\vec{x})\}\right|^{2}
$$

which corresponds to an Airy function (see Fig 1b) and is also known as the diffraction-limited PSF [2], [4]. In practice an AO system tries to estimate $\widehat{\phi} \approx \phi$ such that the residual phase error [5], [6]

$$
\phi_{\text {res }}(\vec{x})=\widehat{\phi}(\vec{x})-\phi(\vec{x})
$$

is minimum and the resulting phase corrected PSF has the form:

$$
p_{\widehat{\phi}-\phi}(\vec{x})=\left|\mathcal{F}^{-1}\{P(\vec{x}) \exp [i(\widehat{\phi}-\phi)(\vec{x})]\}\right|^{2} .
$$

It is evident from the above equation that as $\widehat{\phi}$ tends to $\phi$ (with AO correction) one can achieve $p_{\widehat{\phi}-\phi} \approx p_{0}$ and thus a diffraction-limited image [2], [4] (i.e. limited only by the optical quality of the telescope with the effect of atmosphere optical system completely removed). Correct estimate of $\phi$ is therefore important in eliminating the phase distortion present in the wavefront and thereby recovering the true image of the spatial object. Wavefront phase reconstruction has therefore been an area of active research [7], [1], [8], [9], [10], [11] in ground-based astronomical imaging.

In principle, in an AO system, $\phi$ is estimated as follows : a WFS (generally a Shack-Hartmann WFS [2]) records $\phi$ in the form of its low-resolution slope (gradient) measurements. Given these measurements, a controller generates correction signals (corresponding to phase values) of the wavefront. These signals are then applied as high voltages (after passing through Digital-toAnalog converters) to actuators placed beneath a deformable mirror (DM). The actuators then push or pull the DM to update the shape of the mirror, thereby creating a counter wavefront $\widehat{\Psi}$ according to the shape of the incident wavefront $\Psi$ (see equation (1)). The whole process is repeated iteratively until $\phi_{\text {res }}$ (see equation (4)) is minimum. The process of recreating $\phi$ from the WFS measurements (carried out by the controller) is generally expressed in a matrix-algebra framework [7], [1] as :

$$
\mathrm{g}=\Gamma \Phi+\mathrm{n}
$$

where $\Phi$ is a vector of discretized phase values representing the wavefront phase $\phi$, g represents the slope measurements of the WFS, $\Gamma$ is the discrete differential operator and $\mathrm{n} \sim \mathcal{N}\left(0, \sigma^{2} \mathrm{I}\right)$ is the noise vector. The solution to this problem is that one searches for a $\widehat{\Phi}$ close to $\Phi$, such that it 


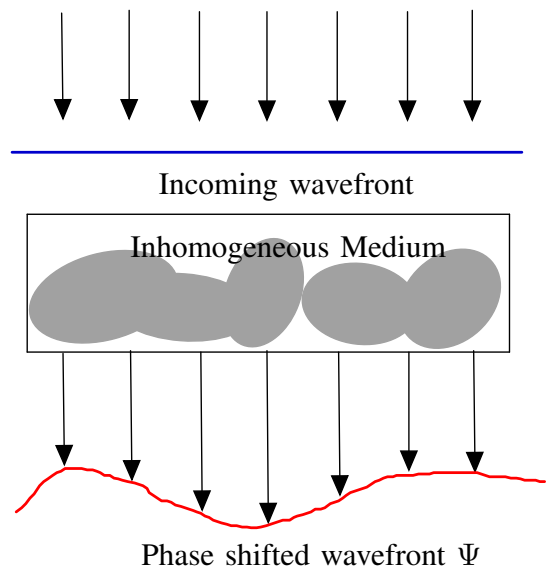

(a)

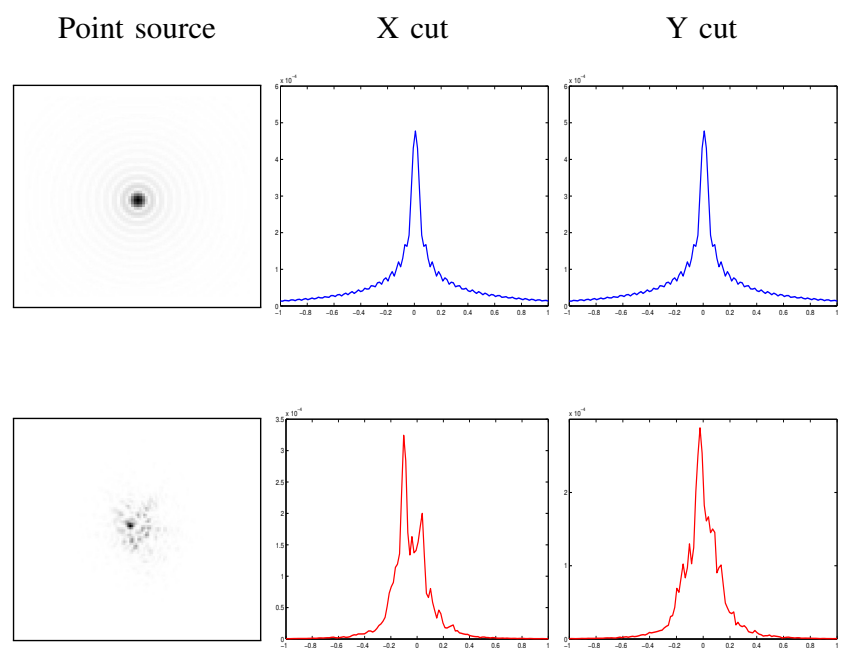

(b)

Fig. 1: (a) The effects of atmospheric turbulence on an incoming spatial wavefront. (b) Effects of turbulence on image formation in ground-based astronomy. Top row (from left to right): simulated negative image of a point source (star) in an ideal telescope without atmosphere (corresponds to an Airy function), the X cut and Y cut of the image. Bottom row (from left to right): simulated negative image showing what a point source (star) would look like through a ground-based telescope in presence of atmosphere (the speckle formation of the image is due to turbulence in the atmosphere), the $\mathrm{X}$ cut and $\mathrm{Y}$ cut of the speckle image.

minimizes the least squares criterion $\underset{\Phi}{\operatorname{argmin}}\|\Gamma \Phi-\mathrm{g}\|_{2}^{2}$ in the $L^{2}$ norm. Solution methods for this problem can be broadly classified into two categories [1], [8] : the maximum likelihood (ML) technique and the maximum a posteriori (MAP) technique. The ML method tries to determine the unknown $\Phi$ such that it maximizes the probability of producing the measurement vector $\mathrm{g}$. The MAP estimator, on the other hand, tries to find the most likely $\widehat{\Phi}$, given the data $\mathrm{g}$ and some prior information on $\Phi$. The ML method, in the case where the statistics of noise is not known, yields to the generalized least squares solution which is the solution classically used to formulate the phase under real-time constraints. The least squares solution is, however, unstable for large-scale AO systems [7], [1], [12] and the solution that is commonly preferred is known as 
the minimum variance estimation technique [7], [1], [11]. The MVW estimator tries to minimize the statistical average of the wavefront phase residual error $\epsilon=\left\langle\|\widehat{\Phi}-\Phi\|^{2}\right\rangle$. The final solution leads to [7], [1]:

$$
\widehat{\Phi}=\left(\Gamma^{T} \Gamma+\sigma^{2} C_{\Phi}^{-1}\right)^{-1} \Gamma^{T} \mathbf{g}
$$

where $\Phi$ is assumed to be Gaussianly distributed with a known covariance matrix $C_{\Phi}$ and $\Gamma^{T}$ is the transpose of $\Gamma$. There are many approaches to solve equation (7) that have been of major interest in recent years. Conjugate gradient (CG) and precondition conjugate gradient (PCG) methods [9], [13], [7], [14] are the most widely used solvers for this purpose, with the use of multigrid as a preconditioner [9], [15] to PCG iterations being the most computationally efficient one.

In this paper, we consider the problem of estimating $\phi$ from the view point of a multifractal system. It has been previously observed and experimentally proved [16], that although the behavior of a wavefront phase distorted due to atmospheric turbulence ${ }^{2}$ can be highly irregular they exhibit certain features that are quite consistent when viewed from different geometric scales. In other words, they exhibit self-similar features while operating at multiple scales. Such multiscale self-similarities are usually observed in the case of multifractal systems [18], [19], [20], [21], [22], where a local power-law governs the behaviour of objects at different scales [19], [20], [23], [24], [25]. This power-law, corresponding to a certain measure of the system, is characterized by values called multifractal exponents, or as it is called in the MMF framework, the singularity exponents (SE) [24]. Previous methods [18], [26] dedicated to the computation of these exponents require ensemble realizations of the same signal (which is not always accessible in practice) and is generally time consuming. In MMF, we design methodologies for precise and pointwise estimate of these exponents for a given realization of the signal. Our phase reconstruction approach is motivated from the idea of extracting the relevant multiscale features of a wavefront phase, through multiresolution analysis [27], [28] (MRA) on the SE (computed on the phase data). Then with the knowledge of the intermediate details (obtained from MRA), we reconstruct the low-resolution Shack-Hartmann gradients to high-resolution. The corresponding

\footnotetext{
${ }^{2}$ Atmospheric turbulence has been known to have effect on light propagation multifractality (in chaotic laser propagation) and star wandering is known to be atleast monofractal [17].
} 
high-resolution phase is then obtained by solving discrete Poisson equation with Neumann boundary conditions.

The paper is organised as follows: in section II we give a brief overview of MMF followed by description of the experimental data in section III. In section IV we introduce our reconstruction technique with the experimantal results discussed in section V. Finally, we conclude in section VI.

\section{MultifRACTAL SYSTEMS AND MMF}

In physics, the multifractal formalism stands as one of the most precise description of systems displaying scale invariant behaviour, these systems having been reported since long time ago, in fact from the early days of statistical mechanics. Scale invariance is related, for instance in high order phase transitions, to power law behaviour of time or spatial correlation functions of intensive physical variables. The exponents of these power laws, called critical exponents, are generalized to critical manifolds which were first described using ordinary fractal sets and their associated dimension. However, it was soon realized that the evolution of some dynamical systems such as turbulent flows involves an intrinsic complexity that cannot be described by a single fractal interface, but rather by a complex arrangement of multifractal nature. In a celebrated paper, Parisi and Frish indicate in their study on turbulence that the different fractal components in a multifractal system are conveniently arranged to give as a result the observed scaling exponents [29]. Since then, the interest in multifractal grew considerably in the physics community. Over the years its description has emerged as a powerful tool for exploiting selfsimilar structures in 2D objects [21], [23], [22], [25], [20], [16]. Multifractal models have proved their merit in different image processing applications ranging from classification [30], [31], [32], [33], segmentation [34], [35], [36], synthesis [37] with some special applications in texture related problems [38], [21], [39].

In the MMF model of mutifractal analysis, we introduce methods to compute localized versions of the SE's (with a focus on overcoming the limitation of computational burden and data extensiveness suffered by previous models). According to the MMF theory, a signal $s(\vec{x})$ is considered to be multifractal if, for at least one functional $\mathbb{T}_{r}$ (depending on the scale $r$ ) and for any point $\vec{x}$, the following equation holds [20], [24]:

$$
\mathbb{T}_{r} s(\vec{x}) \approx \alpha(\vec{x}) r^{h(\vec{x})} \quad(r \rightarrow 0)
$$


where $\alpha(\vec{x})$ is a signal dependent amplitude prefactor. The exponent $h(\vec{x})$ which is a function of the point $\vec{x}$, is called the singularity exponent at point $\vec{x}$. Since $s(\vec{x})$ in our case is $\phi(\vec{x})$, we rephrase equation (8) as:

$$
\mathbb{T}_{r} \phi(\vec{x}) \approx \alpha_{\phi}(\vec{x}) r^{h_{\phi}(\vec{x})} \quad(r \rightarrow 0)
$$

For small number of $r$ 's, the above equation satisfies the equality criteria and the $\mathrm{SE} h_{\phi}(\vec{x})$ can be computed through a log-log regression of equation (9) as :

$$
h_{\phi}(\vec{x})=\lim _{r \rightarrow 0} \frac{\log \left(\mathbb{T}_{r} \phi(\vec{x}) / \alpha_{\phi}(\vec{x})\right)}{\log (r)},
$$

The choice of the functional $\mathbb{T}_{r}$ plays an important role in estimating the SE. For our case, and for the case of determining multiscale feature (ex. edges in the case of natural images [16]) consistency across scales, choosing $\mathbb{T}_{r}$ as the wavelet projection over the norm of the gradients of $s(\vec{x})$ gives excellent results. The preferred wavelet of choice is the $\beta$-Lorentzian wavelet :

$$
\psi(\vec{x})=\psi^{\beta}(\vec{x})=\frac{1}{\left(1+|\vec{x}|^{2}\right)^{\beta}} \quad(\text { for } \beta=1,2,3,4),
$$

which is an isotropic wavelet and therefore do not privilege any particular direction [23]. The chosen functional, denoted by $\mathbb{T}_{\psi} \phi(\vec{x}, r)$, is therefore defined as :

$$
\mathbb{T}_{\psi} \phi(\vec{x}, r)=\int\|\nabla \phi\|(\vec{y}) \frac{1}{r^{d}} \psi\left(\frac{\vec{x}-\vec{y}}{r}\right) \mathrm{d} \vec{y},
$$

with $d$ as the dimension of the signal domain $(d=2$ in the case of images) and $\psi$ as the wavelet function. Multifractal analysis states that the wavelet projections scale as power-laws in $r$ [19], [23], [24], [39]. For multiple values of $r, r=\left\{r_{0}, r_{1}, \cdots, r_{n}\right\}$, equation (9) can be re-written as:

$$
\begin{aligned}
& \log \left(\mathbb{T}_{\psi} \phi\left(\vec{x}, r_{0}\right)\right)=\log \left(\alpha_{\phi}(\vec{x})\right)+h_{\phi}(\vec{x}) \log \left(r_{0}\right) \\
& \log \left(\mathbb{T}_{\psi} \phi\left(\vec{x}, r_{1}\right)\right)=\log \left(\alpha_{\phi}(\vec{x})\right)+h_{\phi}(\vec{x}) \log \left(r_{1}\right) \\
& \log \left(\mathbb{T}_{\psi} \phi\left(\vec{x}, r_{n}\right)\right)=\log \left(\alpha_{\phi}(\vec{x})\right)+h_{\phi}(\vec{x}) \log \left(r_{n}\right)
\end{aligned}
$$

and can be expressed in the matrix framework as:

$$
\underbrace{\left[\begin{array}{c}
\log \left(\mathbb{T}_{\psi} \phi\left(\vec{x}, r_{0}\right)\right) \\
\log \left(\mathbb{T}_{\psi} \phi\left(\vec{x}, r_{1}\right)\right) \\
\vdots \\
\log \left(\mathbb{T}_{\psi} \phi\left(\vec{x}, r_{n}\right)\right)
\end{array}\right]}_{A}=\underbrace{\left[\begin{array}{cc}
1 & \log \left(r_{0}\right) \\
1 & \log \left(r_{1}\right) \\
\vdots & \\
1 & \log \left(r_{n}\right)
\end{array}\right]}_{B} \times \underbrace{\left[\begin{array}{c}
\log \left(\alpha_{\phi}(\vec{x})\right) \\
h_{\phi}(\vec{x})
\end{array}\right]}_{Y} .
$$




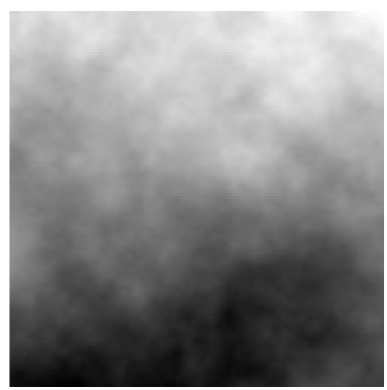

(a)

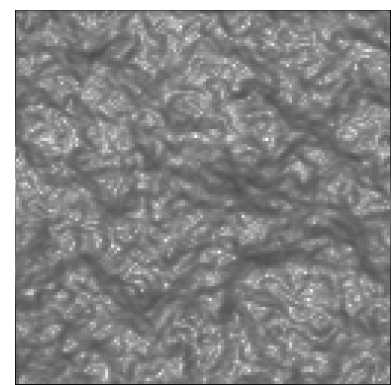

(b)

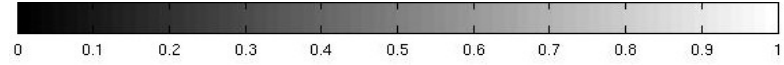

Fig. 2: (a) Image (size : $128 \times 128$ pixels) of a simulated optical phase $\phi$ perturbated by atmospheric turbulence. (b) Image of the singularity exponents (SE) computed on the phase data using $\beta$-Lorentzian wavelet.

Equation (10) can then be solved using the least squares approach:

$$
Y=\left(B^{T} B\right)^{-1} B^{T} A,
$$

with $Y(2)=h_{\phi}(\vec{x})$. The scale $r$ are chosen as dyadic representation of the signal $\phi(\vec{x})$. In Fig 2, we show an example phase data and the SE computed over it using the described procedure.

\section{DESCRIPTION OF DATA}

The data used in our work are datasets of simulated turbulent optical phase generated at ONERA. Data consists of 1000 occurences of turbulent phases defined on a pupil support, corresponding to the following imaging characteristics:

- diameter of the telescope: $8 \mathrm{~m}$,

- seeing at 0.5 microns: 0.85 arcseconds,

- Freed parameter $r_{0}$ at imaging wavelengths: $70 \mathrm{~cm}$,

- $\frac{D}{r_{0}}=11.4$,

- wind's speed: $12.5 \mathrm{~m} / \mathrm{s}$. Taylor's hypothesis is used: translation of the phase along $x$-axis,

- acquition frequency: $200 \mathrm{~Hz}$.

The pupil is defined on $256 \times 256$ pixels. Data is generated in the FITS format [40]. For the statistical work shown in the section $\mathrm{V}$ we need a set of appropriate sub-images, which 


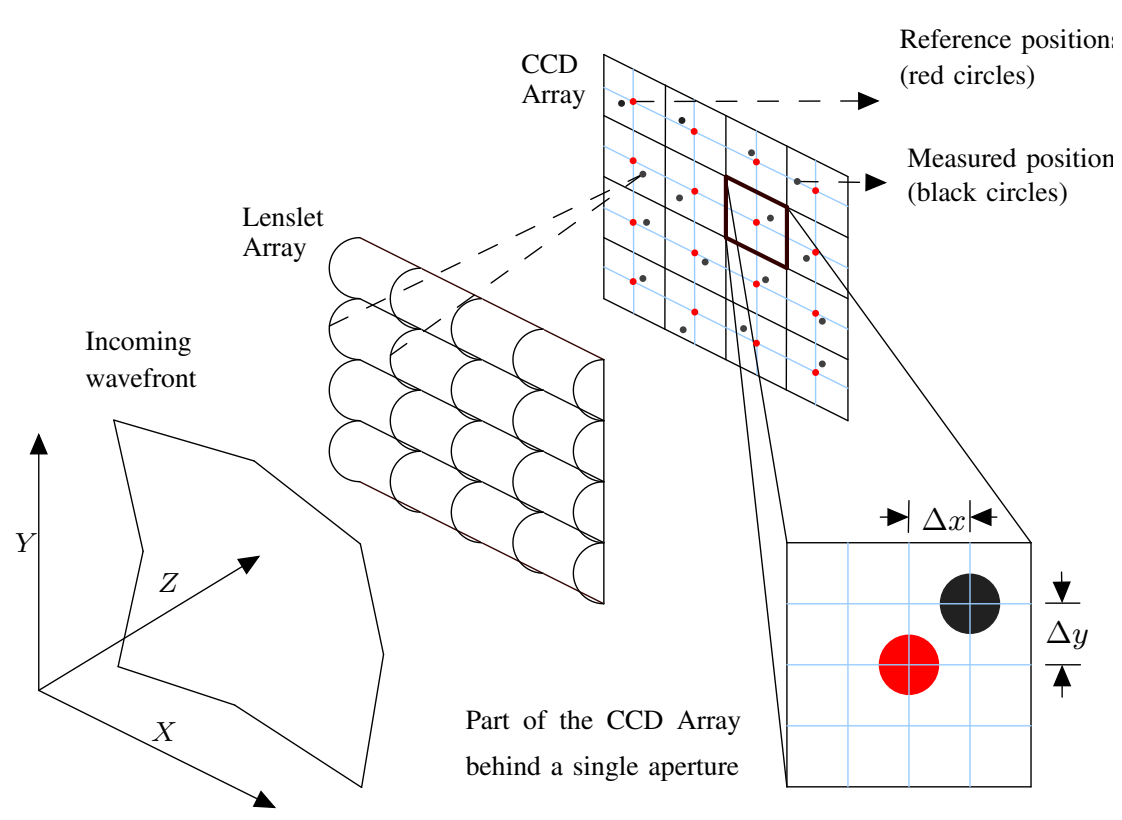

(a)

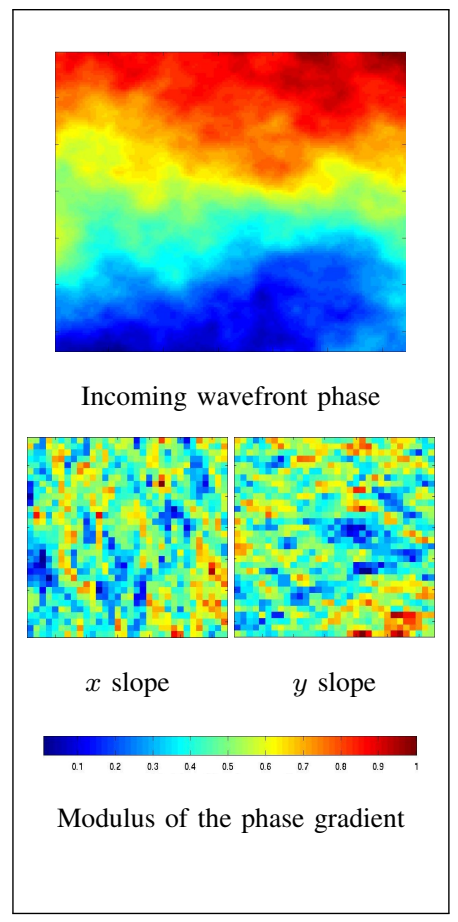

(b)

Fig. 3: (a) Schematic representation of a Shack-Hartmann (SH) wavefront sensor. An incident wavefront travelling along the $Z$-axis, after entering the telescope pupil, is sampled by an array of lenses (called lenslet array), and forms an image of the source on the CCD array. If the wavefront is planar, each lenslet forms an image of the source at its focus (marked by red). If the wavefront is distorted, the images are shifted from their reference position (marked by black). This shift in position is proportional to the mean slope of the wavefront and can be measured with equation (16). (b) Top row: Phase $\phi$, of an incident turbulent wavefront $\Psi$, used for our experiments. Bottom row (from left to right): SH acquisition of the $x$ slope and $y$ slope for the phase data. Displacement of image centroids in two orthogonal directions $x, y$ are proportional to the average gradients over the CCD array sub-apertures.

must be, due to the requirements imposed by dyadic wavelet analysis, square sampled with a sampling size being a power of 2 (because we use a dyadic multiresolution algorithm). To avoid sub-reconstruction and Gibbs phenomena coming from the strong transition associated to the pupil's boundary, we take a sub-image made of $128 \times 128$ pixels centered in the middle of the pupil in the original data. The WFS slope measurements $g$ (refer to equation (6)) are calculated 
from these given sub-images. In this context, a short overview of the principle of operation of a Shack-Hartmann (SH) WFS will help us in understanding how the slope measurements are done.

\section{A. Principle of operation of a SH WFS}

The principle of operation of a SH WFS is explained in Fig 3a. In a SH sensor, an array of lenses (also called a lenslet array) is placed in a conjugate pupil plane at the entrance of the telescope. Each lenslet covers a small part of the aperture (or pupil), the area covered is known as the sub-aperture area. A wavefront incident on the telescope pupil, is sampled by these lenslets and an image of the source is formed on a detector ${ }^{3}$, placed in the focal plane of the lenslet array. When the wavefront is plane, each lenslet forms an image of the object (source) at its focus. But, in general, due to turbulence when the wavefront gets distorted, each lenslet sees a tilted version of the wavefront and the corresponding images are shifted from their reference position [8], [2], [12]. This shift in position is proportional to the mean slope of the wavefront and therefore can be measured [12]. The slopes measured by a SH WFS are proportional to the gradient of $\phi$ averaged over the sub-aperture area $S$ [2], [12], and can be written as:

$$
\left\{\begin{array}{c}
\mathrm{g}_{x}=\frac{\lambda}{2 \pi S} \int_{S} \frac{\partial \phi}{\partial x} \mathrm{~d} x \mathrm{~d} y+n_{x} \\
\mathrm{~g}_{y}=\frac{\lambda}{2 \pi S} \int_{S} \frac{\partial \phi}{\partial y} \mathrm{~d} x \mathrm{~d} y+n_{y}
\end{array}\right.
$$

where $\lambda$ is the central wavelength of the detector; $n_{x}$ and $n_{y}$ takes into account any type of noise associated with the WFS measurements.

We therefore compute the slopes as follows : from the given $\phi$ 's, we compute their gradient and produce an averaged gradient over a window of size $4 \times 4$ pixels, normalized by the size of the window (16 square pixels) thus resulting in a $32 \times 32$ pixels sub-image corresponding to the $x$ and $y$ slope measurement (i.e. $\mathrm{g}=\left[\mathrm{g}_{x} \mathrm{~g}_{y}\right]$ of equation (6)) of a SH WFS. For our experimental purpose, we have also generated gradients of size $64 \times 64$ pixels by the same procedure, normalized by their respective window size (i.e. $2 \times 2$ pixels). In Fig 3 b, we show

\footnotetext{
${ }^{3}$ The detector can be a four quadrant detector for each sub-aperture or a charged-coupled device (CCD) [1].
} 
a high-resolution phase-screen $\phi$ and its corresponding low-resolution $32 \times 32$ pixels $x$ and $y$ slope measure $\mathrm{g}$, which we have used for experimental demonstration in this paper.

\section{WAVEFront Phase Reconstruction}

The aim of our reconstruction algorithm is, given the low-resolution gradient measurements $\mathrm{g}_{x}, \mathrm{~g}_{y}$, we will try to reconstruct a high-resolution phase $\widehat{\phi} \approx \phi$ by taking into account an $a$ priori information of $\phi$. The a priori information that we use is computed as follows : given $\phi$, we compute $h_{\phi}$ using equation (15), which serves as input to our reconstruction algorithm. In real-time AO correction, however, the classical reconstruction algorithms [7], [8], [9], [10] estimate $\phi$ from the WFS measurements $\mathrm{g}_{x}, \mathrm{~g}_{y}$, without taking into account any knowledge of the true phase $\phi$. We therefore, first, validate the potential of our algorithm with knowledge of $h_{\phi}$ obtained from $\phi$ and then we move on to a more practical approach, where we don't take into account any information of $\phi$. Rather what we use, as $\phi$, is a fixed simulated FFT based phase-screen, say $\phi_{f t}$, obtained by McGlammery method [41] (see section VII) satisfying the laws of atmospheric turbulence. From this $\phi_{f t}$, we compute $h_{\phi_{f t}}$ which then serves as the $a$ priori information.

Our reconstruction algorithm is a two step process: analysis consists in computing a MRA on $h_{\phi}$, with a choosen wavelet, and extract the details. Synthesis consists in using these details to obtain gradients at higher resolution, from which the phase is estimated. In this context, a brief summary of MRA and wavelet transform will be helpful to understand the undergoing process.

\section{A. Multiresolution Analysis (MRA) \& wavelet transform}

Multiresolution analysis is a mathematical formalism that deals with the phenomenon of detailstructured viewing of objects [27]. Data redundancy is minimized by the use of Hilbertian frames such as the one obtained by the use of dyadic wavelets [28].

The analysis part using MRA associated to a fast wavelet transform [28] decomposes successively each approximation image (shown as $\alpha_{j}^{0}$ in Fig 4) into a coarser approximation $\left(\alpha_{j+1}^{0}\right)$ plus the wavelet coefficients (i.e. details, shown as $\alpha_{j+1}^{1}, \alpha_{j+1}^{2}$ and $\alpha_{j+1}^{3}$ in Fig 4). Every level of decomposition gives rise to an image fourth smaller than the previous one (as shown in Fig 4). The process can be mathematically formulated by representing a given signal $s(\vec{x})$ in a dyadic 


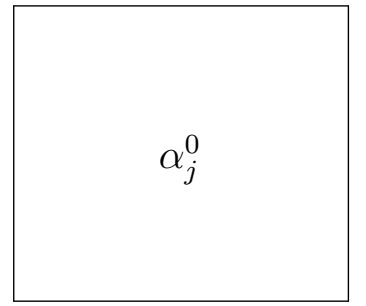

Original image

\begin{tabular}{|c|c|}
\hline$\alpha_{j+1}^{0}$ & $\alpha_{j+1}^{1}$ \\
\hline$\alpha_{j+1}^{2}$ & $\alpha_{j+1}^{3}$ \\
\hline
\end{tabular}

Level 1 decomposition

\begin{tabular}{|c|c|}
\hline \begin{tabular}{l|l}
$\alpha_{j+2}^{0}$ & $\alpha_{j+2}^{1}$ \\
\end{tabular} & $\alpha^{1}$ \\
\hline$\alpha_{j+2}^{2} \alpha_{j+2}^{3}$ & \\
\hline$\alpha_{j+1}^{2}$ & $\alpha_{j+1}^{3}$ \\
\hline
\end{tabular}

Level 2 decomposition

Fig. 4: Representation of the decomposition in multiresolution analysis (MRA). « $\alpha_{j+1}^{0} »$ is the approximation of the initial image $\left\langle\alpha_{j}^{0} »\right.$ at the resolution $j+1 .\left\langle\alpha_{j+1}^{1} »,\left\langle\alpha_{j+1}^{2} »\right.\right.$ and $\left\langle\alpha_{j+1}^{3} »\right.$ are the horizontal, vertical and diagonal details respectively at the resolution $j+1$.

wavelet basis of mother wavelet $\psi^{i}$ [28]. The wavelet coefficients $\alpha_{j, k}^{i}$ can be obtained by a simple projection of the signal onto $\psi_{j, k}^{i}$ as:

$$
\alpha_{j, k}^{i}=\int \psi_{j, k}^{i}(\vec{x}) s(\vec{x}) \mathrm{d} \vec{x}
$$

where the basis function $\psi_{j, k}^{i}(\vec{x})=2^{-j} \psi^{i}\left(2^{-j} \vec{x}-k\right)$ (also called the wavelet function) represents the contribution to the signal $s(\vec{x})$ due to a basic feature of orientation $i$ ( $i=0$ represents the approximation of the image (i.e. $\alpha_{j}^{0}$ ) at the resolution $j, i=1$ represents the horizontal details $\alpha_{j}^{1}, i=2$ the vertical details $\alpha_{j}^{2}$, and $i=3$ the diagonal details $\alpha_{j}^{3}$ ) at the dyadic scale $2^{j}$ and at the position $2^{j} k$ [27] (see Fig 4).

Reconstruction of the signal $s(\vec{x})$ from the wavelet coefficients finds each $\alpha_{j}$ from $\alpha_{j+1}^{i}$ and is expressed as [28]:

$$
s(\vec{x})=\sum_{i=0,1,2,3} \sum_{j} \sum_{k} \alpha_{j, k}^{i} \psi_{j, k}^{i}(\vec{x}),
$$

which is the synthesis of a signal in the Hilbertian formulation with wavelet projection operators [28] and forms the backbone of our second step, the synthesis part.

\section{B. Reconstruction technique}

Our reconstruction approach is summarized accordingly :

- We first compute $h_{\phi}(\vec{x})$ using equation (15). We consider $h_{\phi}(\vec{x})$ as the approximation image $\alpha_{j}^{0}$ (Fig 4)) at the resolution scale $j$ (here $128 \times 128$ pixels). 
- A MRA on $h_{\phi}(\vec{x})$ gives rise to an approximation image $\left(\alpha_{j+1}^{0}\right)$ and the details i.e. the wavelet coefficients $\alpha_{j+1}^{1}, \alpha_{j+1}^{2}$ and $\alpha_{j+1}^{3}$ at the next coarser scale $j+1$.

- Every level gives rise to an image fourth smaller than the previous one. We repeat the operation 2 times to an approximation $\alpha_{j+2}^{0}$ of size $32 \times 32$ pixels. We store the details of the details obtained in the intermediate levels.

- This part of the operation is the called the analysis part of our algorithm.

- The next part of the operation is called the synthesis part.

- We replace the $\alpha_{j+2}^{0}$ image (obtained from the analysis operation) with $\mathrm{g}_{x}$ and $\mathrm{g}_{y}(32 \times 32$ pixels, see section III).

- We then separately reconstruct $\mathrm{g}_{x}$ and $\mathrm{g}_{y}$, to their desired high-resolution (i.e. $128 \times 128$ pixels) using the intermediate details (refer equation (18)) stored during the analysis part.

- $\widehat{\phi}$ is then estimated from the high-resolution reconstructed versions of $\mathrm{g}_{x}, \mathrm{~g}_{y}$ using a fast Poisson solver [42].

We have tested our reconstruction algorithm with 37 standard wavelets, belonging to different families, as the wavelet of choice for the MRA. The best results, in terms of reconstruction, are obtained with the Battle-Lemarié wavelet of order 3.

\section{RESULTS AND DISCUSSION}

Reconstruction has been done for all the 1000 phase-screens $(\phi)$ provided by ONERA, with gradients of two different sizes $(32 \times 32$ pixels and $64 \times 64$ pixels respectively). The gradients are computed as discussed in section III-A. The experiments are repeated after adding different levels of Gaussian white noise to $\phi$ and then computing $\mathrm{g}_{x}$ and $\mathrm{g}_{y}$ from it.

\section{A. Model validation with known phase $\phi$}

As discussed in the previous section, we check the performance of our reconstruction algorithm, with $h_{\phi}$ as input to the analysis part of the algorithm. For demonstration purpose in this paper, we show the reconstruction results for a single $\widehat{\phi}$, under different levels of signal-to-noise ratio (SNR) in Table I. Comparison between the power spectral density (PSD) of $\widehat{\phi}$ and $\phi$, shown in Table I, shows similar behavior of the curves and confirms the superior quality of reconstruction. Quantitative evaluation of the reconstruction quality is done using the following 
TABLE I: Results: A reconstructed phase $\widehat{\phi}$. Row 1: $\widehat{\phi}$ under different levels of signal-to-noise ratio (SNR). Row 2: Comparison of the power spectral density (PSD) between the true phase $\phi$ and $\widehat{\phi}$ under different levels of SNR.

No noise

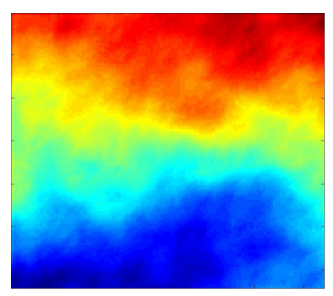

$\mathrm{SNR}=40 \mathrm{~dB}$
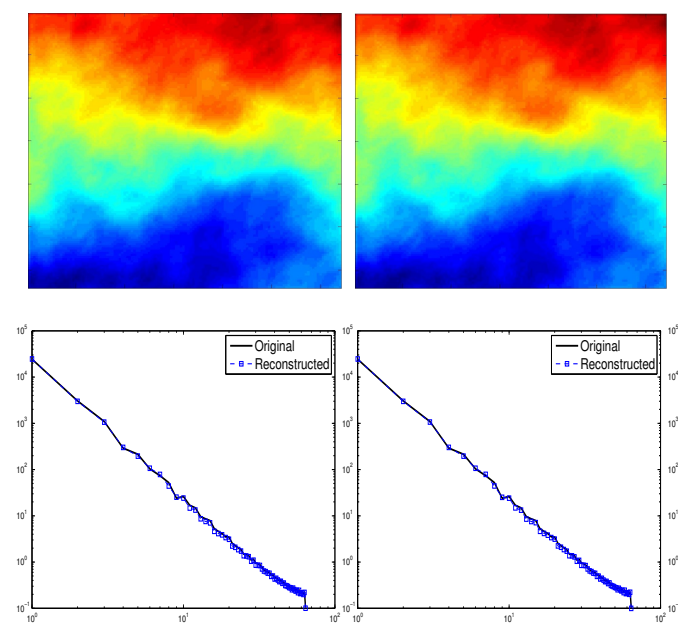

$\mathrm{SNR}=20 \mathrm{~dB}$
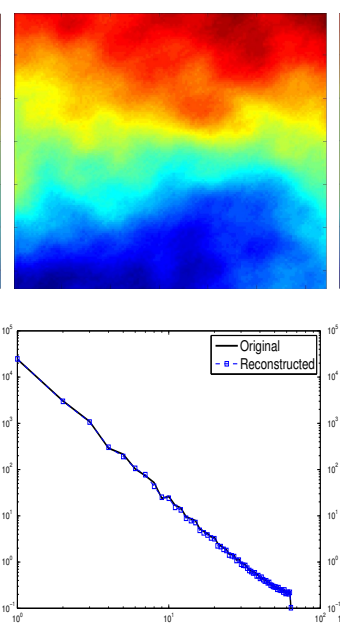

$\mathrm{SNR}=14 \mathrm{~dB}$
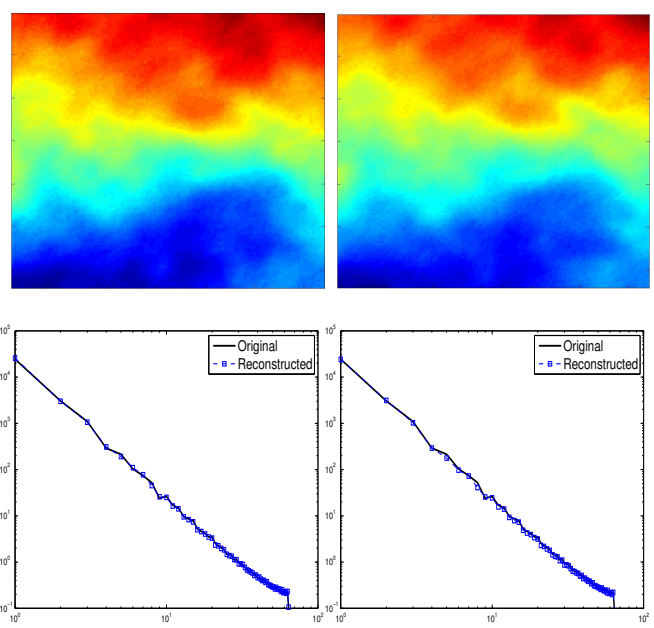

$\mathrm{SNR}=6 \mathrm{~dB}$

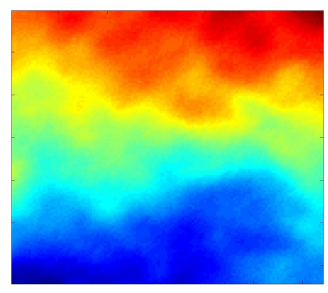

- Power spectral density : True Phase

- - Power spectral density : Reconstructed Phase

TABLE II: Quantitative evaluation of $\widehat{\phi}$ (for Table I) using root-mean-square error (RMSE) and peak signal-to-noise ratio (PSNR, expressed in decibels $\mathrm{dB}$ ) metrics (see equation 19 and equation 20).

\begin{tabular}{|c|c|c|c|c|c|}
\hline SNR & No noise & $40 \mathrm{~dB}$ & $20 \mathrm{~dB}$ & $14 \mathrm{~dB}$ & $6 \mathrm{~dB}$ \\
\hline RMSE & 0.0143 & 0.0187 & 0.0188 & 0.0209 & 0.0288 \\
\hline PSNR & 29.62 & 29.22 & 29.20 & 28.75 & 28.37 \\
\hline
\end{tabular}

image-quality metrics: (i) root-mean-square error (RMSE) and (ii) peak signal-to-noise ratio (PSNR, expressed in decibels $\mathrm{dB}$ ), defined as:

$$
\mathrm{RMSE}=\sqrt{\frac{\sum_{n, m}|\phi(\vec{x})-\widehat{\phi}(\vec{x})|^{2}}{n \times m}}
$$


TABLE III: Results : Point spread function (PSF). The X cut (row 1) and Y cut (row 2) of the PSF for $\widehat{\phi}$ (under different levels of SNR) are displayed in red dashed lines and diamond signs, the black solid line curve corresponds to the $\mathrm{X}$ cut (row 1) and $\mathrm{Y}$ cut (row 2) of the true PSF. The blue dashed line curve is the absolute difference of the two curves. The $y$-axis corresponds to the square of the normalized image plane irradience and the $x$-axis corresponds to the angular distance in arseconds.

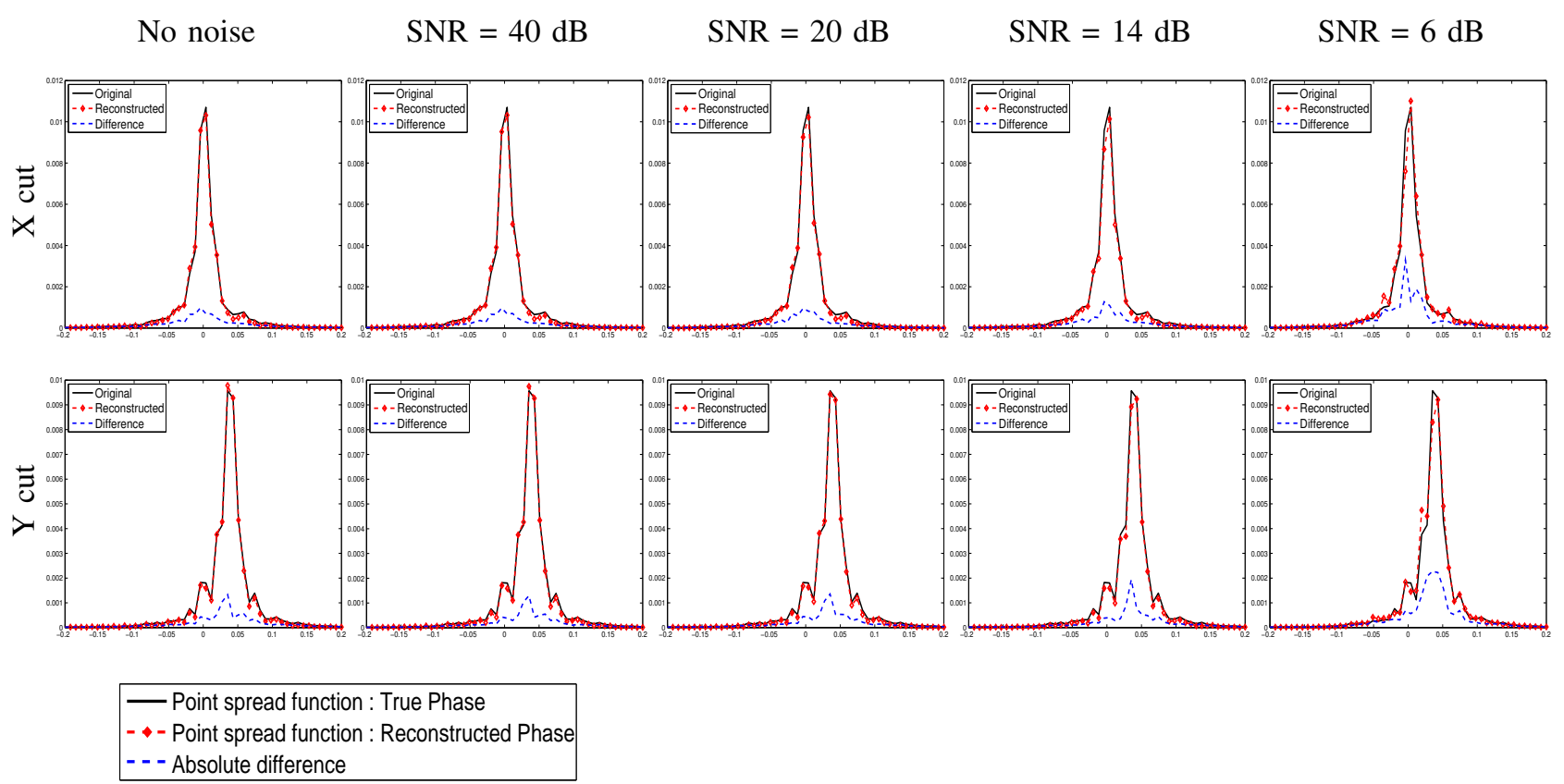

$$
\operatorname{PSNR}=20.0 \times \log _{10} \frac{\max (\phi(\vec{x}))}{\operatorname{RMSE}}
$$

where $n \times m$ is the size of a phase-screen. The results are shown in Table II. Generally PSNR values within a range of $20 \mathrm{~dB}$ to $40 \mathrm{~dB}$ are considered 'good', with anything above 40 $\mathrm{dB}$ is considered near accurate. The corresponding RMSE values will be between 0.10 to 0.01 respectively (this important topic is, for instance, developed in other articles, e.g. ref [26]).

We further validate the quality of our reconstruction, in terms of the PSF and the optical transfer function (OTF), which is essentially the Fourier transform of the PSF. In Table III and Table IV, we compare the X cut and Y cut of the PSF and modulus of the OTF (|OTF $\mid$ ) between 
TABLE IV: Results : Modulus of OTF ( $|\mathbf{O T F}|$ ). The X cut (row 1) and Y cut (row 1) of $|\mathrm{OTF}|$ for $\widehat{\phi}$ (under different levels of SNR) are displayed with a logarithmic scale in red dashed lines, the black solid line curve corresponds to the X cut (row 1) and Y cut (row 2) of the true $|\mathrm{OTF}|$ and the blue dashed line curve corresponds to the absolute difference of the two curves. The $y$-axis corresponds to the logarithm of the $|\mathrm{OTF}|$ and the $x$-axis represents the normalized frequency in $D / \lambda$ units.
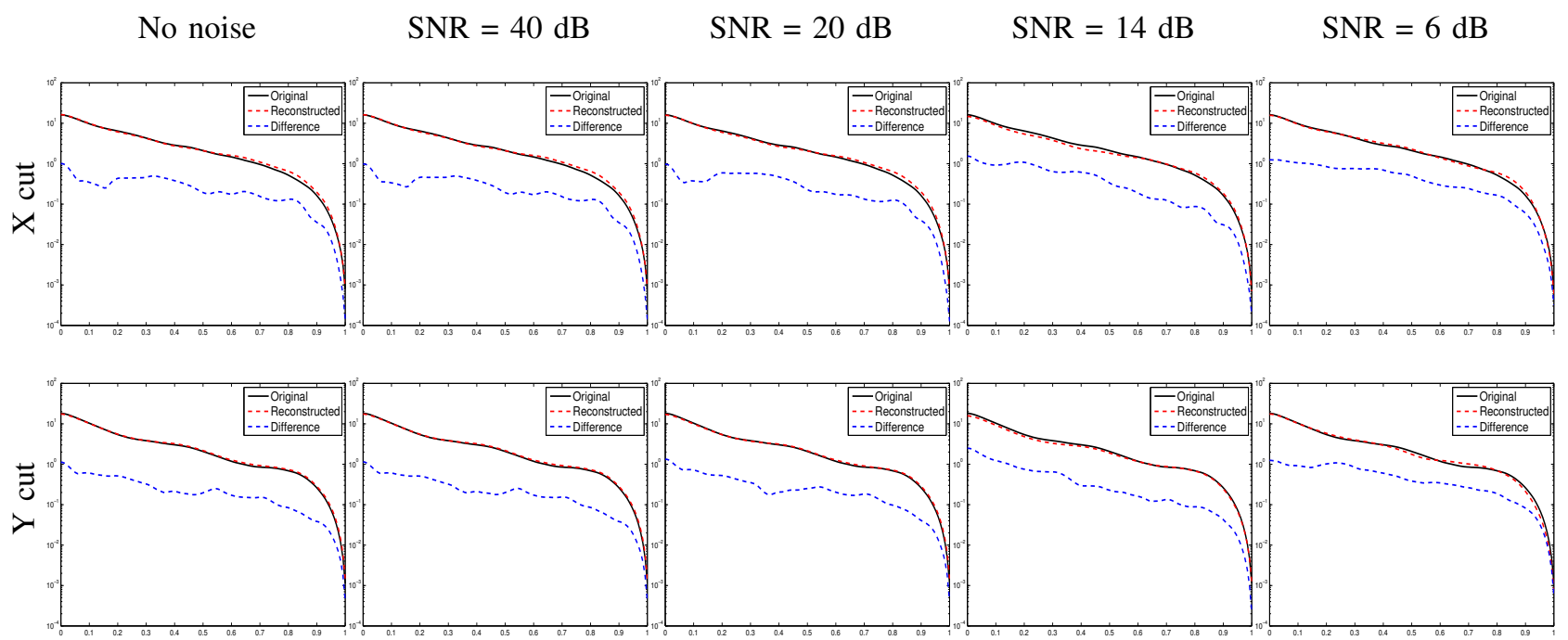

- Modulus of the OTF : True Phase
- - - Modulus of the OTF : Reconstructed Phase
- - Absolute difference

the true phase $\phi$ and $\widehat{\phi}$. Here also, we see the similar behaviour of the curves (shown in black and red for $\phi$ and $\widehat{\phi}$ respectively) under different levels of SNR, with very low error (represented by the blue curve) between them. The results further validate the high quality of reconstruction.

We then move on to compare the reconstruction quality of our method with the output obtained from the following classical linear solvers:

- MVW estimator using multigrid preconditioned conjugate gradients (Multigrid PCG) [7], [9],

- MVW estimator using conjugate gradients (CG) [7],

- Least squares estimation [8] and

- Exponential estimation [10]. 
TABLE V: Quantitative evaluation of the performance of different phase reconstruction algorithms. The RMSE and PSNR (in dB) values are the average values computed for 1000 $\widehat{\phi}$.

\begin{tabular}{|c|c|c|c|c|c|c|}
\hline & \\
\hline & & SNR & $40 \mathrm{~dB}$ & $26 \mathrm{~dB}$ & $14 \mathrm{~dB}$ & $6 \mathrm{~dB}$ \\
\hline \multirow{4}{*}{ Gradient size: } & \multirow{2}{*}{ MMF (proposed) } & PSNR & 32.59 & 32.52 & 32.43 & 30.07 \\
\hline & & RMSE & 0.0091 & 0.0093 & 0.0095 & 0.0130 \\
\hline & & PSNR & 32.08 & 31.33 & 30.21 & 28.56 \\
\hline & & RMSE & 0.0103 & 0.0123 & 0.0158 & 0.0232 \\
\hline \multirow[t]{6}{*}{$64 \times 64$ pixels } & \multirow{2}{*}{ MVW (CG) [7] } & PSNR & 32.04 & 31.31 & 30.19 & 28.52 \\
\hline & & RMSE & 0.0105 & 0.0123 & 0.0160 & 0.0235 \\
\hline & \multirow{2}{*}{ Least squares [8] } & PSNR & 32.06 & 31.32 & 30.19 & 28.53 \\
\hline & & RMSE & 0.0104 & 0.0123 & 0.0158 & 0.0234 \\
\hline & \multirow{2}{*}{ Exponential [10] } & PSNR & 31.30 & 30.48 & 29.39 & 27.68 \\
\hline & & RMSE & 0.0126 & 0.0148 & 0.0193 & 0.0235 \\
\hline \multirow{4}{*}{ Gradient size: } & \multirow{2}{*}{ MMF (proposed) } & PSNR & 30.01 & 29.88 & 28.84 & 27.93 \\
\hline & & RMSE & 0.0125 & 0.0176 & 0.0214 & 0.0265 \\
\hline & & PSNR & 29.92 & 29.42 & 28.59 & 27.20 \\
\hline & 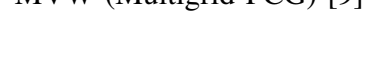 & RMSE & 0.0170 & 0.0190 & 0.0230 & 0.0317 \\
\hline \multirow[t]{6}{*}{$32 \times 32$ pixels } & \multirow{2}{*}{ MVW (CG) [7] } & PSNR & 29.88 & 29.41 & 28.58 & 27.18 \\
\hline & & RMSE & 0.0171 & 0.0190 & 0.0230 & 0.0318 \\
\hline & \multirow{2}{*}{ Least squares [8] } & PSNR & 29.91 & 29.40 & 28.57 & 27.14 \\
\hline & & RMSE & 0.0170 & 0.0191 & 0.0232 & 0.0322 \\
\hline & \multirow{2}{*}{ Exponential [10] } & PSNR & 29.33 & 28.79 & 27.83 & 26.30 \\
\hline & & RMSE & 0.0195 & 0.0221 & 0.0276 & 0.0393 \\
\hline
\end{tabular}

The results of reconstruction using gradients of size $64 \times 64$ pixels and $32 \times 32$ pixels, under different levels of SNR, are presented in Table V. We show the averaged PSNR and RMSE values for the $1000 \widehat{\phi}$ 's in Table V. Results show that the proposed method for phase reconstruction exceeds over the conventional methods, in terms of quality. 
TABLE VI: Reconstruction performance, in terms of residual phase PSD, when true phase $\phi$ is unknown. Here we use a fixed FFT phase-screen $\phi_{f t}$ (generated using McGlammery method [41], see section VII) as a substitute of $\phi$ for our reconstruction algorithm. The $\widehat{\phi}$ thus obtained are then compared with those obtained from a MVW estimator, in terms of their residual phase ( $\phi_{\text {res }}$, see equation 4) power spectral density (PSD) plotted against spatial frequency in the logarithmic scale. The red dashed line and box sign represents the average $\phi_{\text {res }}$ (for $1000 \widehat{\phi}$ ) obtained from the proposed reconstruction (MMF), the red solid line represents the average $\phi_{\text {res }}$ of MVW reconstruction. We also plot the average PSD of $\phi$, shown in black dashed line and triangle sign (true phase), as a reference line to better illustrate the level of correction achieved by both the reconstructors. Reconstruction is done on two different set of gradients. Row 1: $\widehat{\phi}$ from gradients of size $64 \times 64$ pixels. Row 2: $\widehat{\phi}$ from gradients of size $32 \times 32$ pixels.
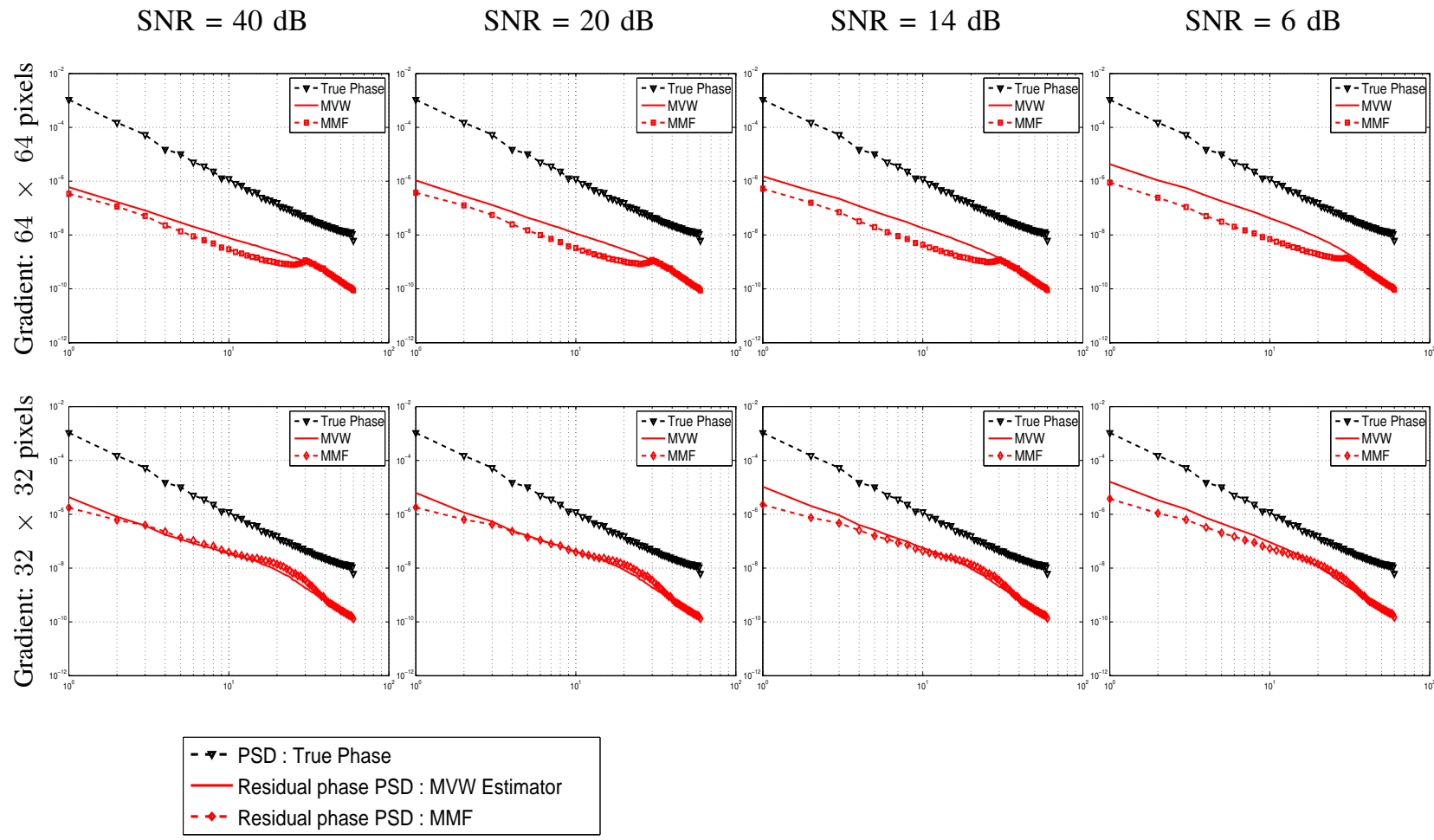

\section{B. Comparison of the residual phase PSD}

We now move on to validate our reconstruction algorithm in a more practical scenario, where we don't have any information of the high-resolution phase $\phi$ but only the SH-WFS measure- 
TABLE VII: Reconstruction performance, in terms of residual phase PSD, when true phase $\phi$ is known. Same legend as for Table VI.
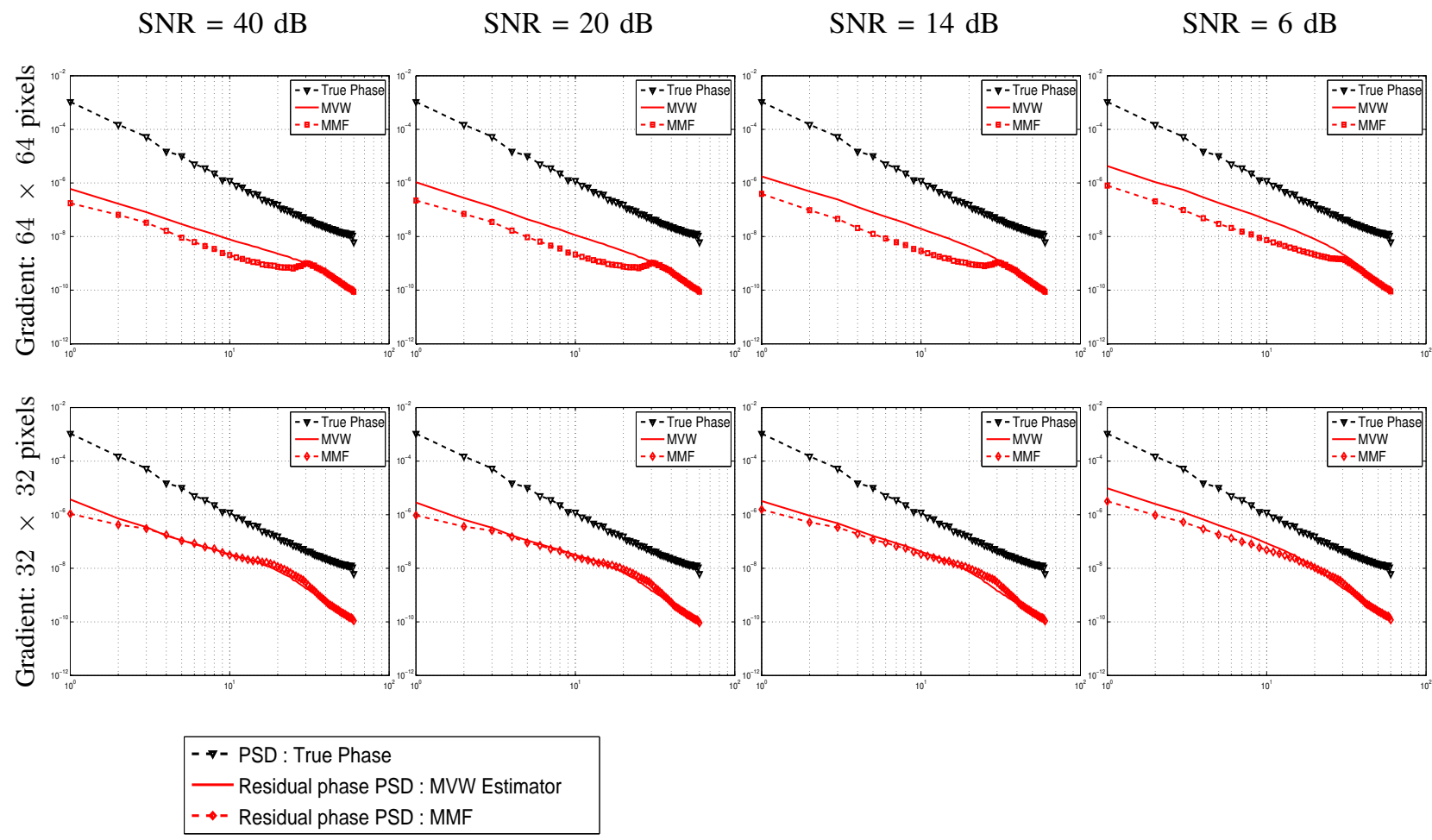

ments $\mathrm{g}_{x}$ and $\mathrm{g}_{y}$. In this case, we generate a fixed FFT phase-screen $\phi_{f t}$ (using McGlammery method [41], see section VII) and use it as a substitute of $\phi$. We compute the SE over $\phi_{f t}$, say $h_{\phi_{f t}}$ and use it as input to the analysis part of our algorithm. The results thus obtained are then compared with those obtained from a MVW estimator, in terms of their residual phase ( $\phi_{\text {res }}$, see equation 4) power spectral density (PSD) plotted against spatial frequency in the logarithmic scale. The lower the value of $\phi_{\text {res }}$, the higher the quality of reconstruction, as is clearly evidenced in Table VI, where red dashed line and box sign represents the average $\phi_{\text {res }}$ (for $1000 \widehat{\phi}$ ) obtained from the proposed reconstruction, the red solid line represents the average $\phi_{\text {res }}$ of MVW reconstruction. We also plot the average PSD of $\phi$, shown in black dashed line and triangle sign in Table VI, as a reference line to better illustrate the level of correction achieved by both the reconstructors. Similar results obtained using $h_{\phi}$ as input, to the analysis part of our algorithm, is shown in Table VII. 
The results clearly show the superiority of our algorithm, compared to the classical MVW estimator, under different levels of SNR (the residual phase error being less in our case). It has also been seen that for the case when reconstruction is made over gradients of size $16 \times 16$ pixels, our method has higher residual error compared to the MVW estimator. The error however reduces considerably as the level of SNR decreases.

For demonstration purpose in this paper, we have shown the comparison with a multigrid PCG [7], [9] based MVW estimator, although we have compared the performance of our reconstruction with CG [7] based MVW estimator, Exponential operator [10] and Least squares estimator [7], [8]. In all the cases the overall performance has been the same, with the performance of our algorithm being superior. It should be noted here, that in practice AO correction is applicable only to the low-frequency correction of the phase components [5], [6] (also called the mirror modes and generally corresponds to the first few zernike polynomials [43]). One is therefore interested in observing the behaviour of the curve, shown in Table VI and Table VII, for the low-frequency regions only.

\section{CONCLUSION}

In this paper, our objective was to introduce a new method to phase reconstruction which is based on computationable microcanonical characterizations of fully developped turbulence in nonlinear physics. This idea has never been exploited before in the community. It allows us to step beyond the classical phase reconstruction methodologies based on linear formulations in a Bayesian framework. In this paper, we therefore show the numerical feasability of our approach, we run it on synthetic turbulent phase-screen used in the simulation for AO testing and we have proposed a methodology which works superior in a noisy environment. The experimental algorithms tested on the synthetic AO data show the same average performance as L2 minimization and pseudo-inverse matrix computation used presently in real AO systems. Implementatation and testing of our method in a real-time AO system, at high real-time frequencies, is the next step and project to achieve and implement.

\section{MCGLAMERY MODEL FOR GENERATING FFT BASED PHASE-SCREENS}

The FFT based phase-screen generation technique proposed by Benjamin. L. McGlammery [41] is widely used, for experimental purposes, due to its simplicity and speed although other models 
do exist [44], [45]. The starting point for the generation of phase map is based on the assumption that atmospheric turbulence follows a Kolmogorov spectrum and has a phase that is statistically uniform over the interval $-\pi$ to $\pi$. One way of describing the phase statistically is by means of its power spectrum. For ground based astronomical applications, the atmospheric phase spectrums can be approximated by the Kolmogorov spectrum :

$$
\Phi(f)=0.033 r_{0}^{-5 / 3} f^{-11 / 3}
$$

which can be easily extended to the Von Kàrmàn spectra:

$$
\Phi(f)=0.033 r_{0}^{-5 / 3} \frac{\exp \left(-\left(f / f_{m}\right)^{2}\right)}{\left(f^{2}+f_{0}^{2}\right)^{11 / 6}}
$$

where $f$ is the spatial frequency, $f_{m}=5.92 / l_{0}$ and $f_{0}=1 / l_{0} . L_{0}$ (the outer scale of turbulence, which has been set to the median Paranal value of $25 \mathrm{~m}$ in our case) and $l_{0}$ (the inner scale, $1 \mathrm{~cm}$ in our case) form the inertial range. $r_{0}$ is known as the fried turbulence parameter $(70 \mathrm{~cm}$ in our case).

The phase-screens are generated as follows:

- A complex array of Gaussian random numbers are generated that serves as the spatial frequency domain for the phase-screen.

- The product of this array with the square root of $\Phi(f)$ is then generated.

- Fourier transform of this product gives rise to the phase-screen.

\section{ACKNOWLEDGMENT}

The work is funded by a CORDIS grant and Région Aquitaine OPTAD research project grant.

\section{REFERENCES}

[1] F. Roddier, Adaptive optics in astronomy, Cambridge University Press, Cambridge, U.K, 1999.

[2] A. Tokovinin, "Cfio overview of adaptive optics," http://www.ctio.noao.edu/ atokovin/tutorial/part3/wfs.html.

[3] D. L. Li and S. Simske, "Atmospheric turbulence degraded image restoration by kurtosis minimization," IEEE Trans. Geosci. Remote Sens. Lett., vol. 13, pp. 244-247, 2008.

[4] M. Shahram and P. Milanfar, "Imaging below the diffraction limit: a statistical analysis," IEEE Trans. Image Process., vol. 5, pp. 677-89, 2004.

[5] J. P. Veran, F. Rigaut, H. Maitre, and D. Rouan, "Estimation of the adaptive optics long-exposure point-spread function using control loop data," J. Opt. Soc. Am. A, vol. 14, pp. 3057-3069, 1997. 
[6] J. P. Veran, F. Rigaut, and H. Maitre, "Adaptive optics long exposure point spread function retrieval from wavefront sensor measurements," in Adaptive Optics, M. Cullum, ed., Vol. 54 of ESO Conference and Workshop Proceedings European Southern Observatory, vol. 54, pp. 497-502, 1995.

[7] J. M. Bardsley, "Wavefront reconstruction methods for adaptive optics systems on ground-based telescopes," SIAM J. Matrix Analysis Applications, vol. 30, pp. 67-83, 2008.

[8] L. Mugnier, G. Le Besnerais, and S. Meimon, Inversion in optical imaging through atmospheric turbulence in Bayesian approach to inverse problems, Jérôme Idier ed., ISTE, John Wiley \& Sons Inc., 2008, ISBN: 878-1-84821-032-5.

[9] L. Gilles, C. R. Vogel, and B. L. Ellerbroek, "A multigrid preconditioned conjugate gradient method for large scale wavefront reconstruction,” J. Opt. Soc. Am. A, vol. 19, pp. 1817-1822, 2002.

[10] D. L. Fried, "Adaptive optics wave function reconstruction and phase unwrapping when branch points are present," Optics Communications, vol. 200, pp. 43-72, 2001.

[11] E. Thiébaut and M. Tallon, "Fast minimum variance wavefront reconstruction for extremely large telescopes," J. Opt. Soc. Am. A, vol. 27, pp. 1046-1059, 2010.

[12] G. Rousset, Wave-front sensing, in Adaptive Optics in Astronomy, F. Roddier, ed., Cambridge University Press, Cambridge, U.K, 1999.

[13] L. Gilles, "Order-n sparse minimum-variance open-loop reconstructor for extreme adaptive optics," Optics Letters, vol. 28, pp. 1927-1929, 2003.

[14] J. A. Fessler and S. D. Booth, "Conjugate-gradient preconditioning methods for shift-variant pet image reconstruction," IEEE Trans. Image Process., vol. 8, pp. 688-99, 2002.

[15] M. D. Pritt, "Phase unwrapping by means of multigrid techniques for interferometric SAR," IEEE Trans. Geosci. Remote Sens., vol. 34, pp. 728-738, 1996.

[16] S. K. Maji and H. M. Yahia, "Edges, transitions and criticality,” Pattern Recognition, vol. 47, pp. $2104-2115,2014$.

[17] L. Zunino, D. Gulich, G. Funes, and A. Ziad, "Experimental confirmation of long-memory correlations in star-wander data," Optics letters, vol. 39, pp. 3718-3721, 2014.

[18] K. Falconer, Techniques in fractal geometry, John Wiley, 1997.

[19] A. Arenodo, E. Bacry, and J. Muzy, "The thermodynamics of fractals revisited with wavelets," Physica A, vol. 213, pp. 232-275, 1995.

[20] A. Turiel and N. Parga, "The multifractal structure of contrast changes in natural images: From sharp edges to textures," Neural Computation, vol. 12, pp. 763-793, 2000.

[21] Y. Xu, X. Yang, H. Ling, and H. Ji, "A new texture descriptor using multifractal analysis in multi-orientation wavelet pyramid," CVPR, 2010.

[22] A. Dziri, C. Goutelard, A. Abdaoui, and H. VU Thien, "A new theoretical approach of wavelet-based multifractal characterization of hf channel scattering function: Theoretical and physical interpretations," IEEE Trans. Geosci. Remote Sens., vol. 50, pp. 1736-1747, 2012.

[23] A. Turiel and A. del. Pozo, "Reconstructing images from their most singular fractal manifold," IEEE Trans. Image Process., vol. 11, pp. 345-350, 2002.

[24] A. Turiel, H. Yahia, and C. J. Pérez-Vicente, "Microcanonical multifractal formalism -a geometrical approach to multifractal systems: Part 1. singularity analysis,” J. Phys. A: Math. Theor., vol. 41, no. 015501, 2008.

[25] D. Charalampidis, T. Kasparis, and W. L. Jones, "Removal of nonprecipitation echoes in weather radar using multifractals and intensity," IEEE Trans. Geosci. Remote Sens., vol. 40, pp. 1121-1131, 2002. 
[26] A. Turiel, C. J. Pérz-Vicente, and J. Grazzini, "Numerical methods for the estimation of multifractal singularity spectra on sampled data: A comparative study," Journal of Computational Physics, vol. 216, pp. 362-390, 2006.

[27] C. Pottier, A. Turiel, and V. Garçon, "Inferring missing data in satellite chlorophyll maps using turbulent cascading," Remote Sensing of Environment, vol. 112, pp. 4242-4260, 2008.

[28] Stéphane Mallat, A Wavelet Tour of Signal Processing, Academic Press, 1999.

[29] U. Frisch, Turbulence, Cambridge Univ. Press, Cambridge MA, 1995.

[30] D. Charalampidis and T. Kasparis, "Wavelet-based rotational invariant roughness features for texture classification and segmentation.," IEEE Trans. Image Process., vol. 11, pp. 825-837, 2012.

[31] S. Deguy, C. Debain, and A. Benassi., "Classification of texture images using multi-scale statistical estimators of fractal parameters.," In BMVC., 2000.

[32] L. M. Kaplan, "Extended fractal analysis for texture classification and segmentation.," IEEE Trans. Pattern Anal. Mach. Intell., vol. 8, pp. 1572-1585, 1999.

[33] J. L. Vehel, P. Mignot, and J. P. Berroir, “Multifractals, texture, and image analysis.," In CVPR, pp. 661-664, 1992.

[34] B. B. Chaudhuri and N. Sarkar, "Texture segmentation using fractal dimension.," IEEE Trans. Pattern Anal. Mach. Intell., vol. 17, pp. 72-77, 1995.

[35] L. Kam and J. Blanc-Talon, "Are multifractal multipermuted multinomial measures good enough for unsupervised image segmentation.," In CVPR, vol. 1, pp. 58-63, 2000.

[36] Y. Xia, D. Feng, and R. Zhao, "Morphology-based multifractal estimation for texture segmentation.," IEEE Trans. Pattern Anal. Mach. Intell., vol. 15, pp. 614-623, 2006.

[37] L. M. Kaplan and C. C. J. Kuo, “Texture roughness analysis and synthesis via extended self similar (ess) model.," IEEE Trans. Pattern Anal. Mach. Intell., vol. 17, pp. 1043-1056, 1995.

[38] G. McGunngile and M. J. Chantler, "Evaluating kube and pentland's fractal imaging model.," IEEE Trans. Image Process., vol. 10, pp. 534-542, 2001

[39] H. Badri, H. Yahia, and K. Daoudi, "Fast and accurate texture recognition with multilayer convolution and multifractal analysis," European Conf. Comp. Vision, 2014.

[40] "The fits format," http://fits.gsfc.nasa.gov/.

[41] B. L. McGlamery, "Computer simulation studies of compensation of turbulence degraded images," in Image Processing. International Society for Optics and Photonics, vol. 74, pp. 225-233, 1976.

[42] A. Agrawal, R. Raskar, and R. Chellappa, "What is the range of surface reconstructions from a gradient field ?," European Conf. Comp. Vision, 2006.

[43] R. J. Noll, “Zernike polynomials and atmospheric turbulence,” J. Optical Society of America, vol. 66, pp. 207-211, 1976.

[44] R. G. Lane, A. Glindemann, and J. C. Dainty, "Simulation of a kolmogorov phase screen.," Waves in Random Media, vol. 2, pp. 209-224, 1992.

[45] J. D. Schmidt, "Numerical simulation of optical wave propagation with examples in matlab.," SPIE Press: Bellingham, WA, 2010. 